\title{
A Study on Rorschach Depression Index in Patients Suffering from Depression
}

\author{
Anwesha Mondal' ${ }^{1}$, Manish Kumar ${ }^{1}$ \\ Department of Psychiatry, N. R. S. Medical College, Kolkata
}

\section{ABSTRACT}

Depression Index (DEPI) is one of the specific Rorschach Index developed as a part of the Comprehensive System. There has been a lot of research on Depression Index which identifies individuals who may be emotionally distraught, cognitively pessimistic, lethargic or self defeating. However, there are evidences which show inconsistent results about the depression index when they are used for the purpose of diagnosis of depressive disorder. The main aim of this paper is to study the Depression Index in patients suffering from moderate and severe level of depression. The sample consisted of 30 patients with depression and 30 normal subjects selected by using purposive sampling technique. Beck Depression Inventory (BDI) was administered on the patient group to assess the severity of depressive symptoms and Rorschach Inkblot Test was administered on patients and normal subjects to assess the structure of the personality. General Health Questionnaire-12 (GHQ) was administered as a screening tool in the group of normal control subjects. Results show difference on Depression Index variables among the two groups. No significant correlation was found between BDI scores and DEPI. The diagnostic efficacy was found to be low for DEPI. In conclusion, it can be said that the DEPI has not been found to be very effective in diagnosing depression.

Key Words : Rorschach Test, Depression Index, depression

\section{INTRODUCTION}

Depression is a state of low mood where the person feels sad, anxious, empty, hopeless, helpless, worthless, guilty, irritable. They often lose interest in activities that were once pleasurable, experience loss ofappetite,lossofsleep, haveproblemsconcentrating, remember-ring details or making decisions, feel reduced energy and may contemplate, attempt

\section{Corresponding Author:}

Anwesha Mondal

Consultant Clinical Psychologist

Email id: mondalanwesha22@yahoo.com or commit suicide. The challenge of identifying variables that might be useful in accurately identifying those cases in which depression is a major issue led to the search for a cluster of variables that would help in discriminating a person suffering from depression than from those who are simply dissatisfied, unhappy and distressed. In this regard Rorschach Inkblot Test has been found to help in clinical assessment. Exner's Comprehensive System includes variables which help in identification of depressive features in general in the form of an index known as Depression Index (DEPI). The DEPI has been held forth as a sensitive and specific indicator of depression diagnoses (Exner, 1993) The 
A Study on Rorschach Depression Index in patients suffering from depression

original DEPI consisted of five variables which was later revised in 1986 on the basis of studies done where it was found that at least 15 variables must be considered if the presence of depressive features is to be identified. These 15 variables form the basis for seven criteria that constitute the revised DEPI. The DEPI contains seven criteria like FV+VF+V/FD responses, color-shading blends/Space responses, egocentricity index, affective ratio/blends responses, sum shading/Sum $C^{\prime}$ responses, MOR responses/ Intellectualization Index, COP responses/Isolation Index which captures parameters like emotional control, emotional expression, self esteem, inferiority feelings, negative thoughts found in the individuals suffering from depression. A score of 5 or more was taken to identify the individuals suffering from depression.

Exner (1991) has reported that an elevated score on the DEPI "correlates very highly with a diagnosis that emphasizes serious affective problems." Likewise, Ganellen (1996a,1996b) has argued that the Rorschach is useful for diagnosing depression. The DEPI did discriminate quite effectively between depressive groups and the three control groups where $70 \%$ of the persons from dysthymic and unipolar groups were correctly identified but the false positive rate among the control groups was considerable ranging from $30 \%$ of non depressed persons and the false negative rate was also unacceptably high (Lipovsky, Finch \& Belter, 1989). Despite such claims, nearly all independent studies have found that the original and revised versions of the DEPI lack sensitivity and are unrelated to diagnoses of depression in either adolescents or adults (Archer \& Krishnamurthy, 1997; Carlson, Kula, \& St. Laurent, 1997; Carter \& Dacey, 1996). However, their findings have been so consistently negative that the overall interpretation is clear. Ball et.al. (1991) evaluated both the original and the revised DEPIs for child and adolescent outpatient $(n=67)$ and inpatient $(n=99)$ samples in order to assess the diagnostic utility of these indices. There were no significant relationships between the original form and the revised form of the DEPI and clinical elevations on the depression scale of the
Personality Inventory for Children in the outpatient sample or treatment team diagnostic judgements in the inpatient sample. These findings sound a strong cautionary note for using only Rorschach Depression indices to diagnose depression in children and adolescents. Carlson (1997) examined the clinical field validity of the Rorschach comprehensive system revised depression index (DEPI) and coping deficit index (CDI). 40 admission protocols from 2 inpatient adult DSM-III-R diagnosed samples (one with Major Depressive Disorder, uncomplicated, and one with Major Depressive Disorder and concurrent Borderline Personality Disorder) were compared. Hypotheses were (a) both groups would be identified by the revised DEPI, and (b) if the Depressed Borderline group was not identified by the DEPI, it would be identified by the CDI. Both hypotheses were negated.

Viglione (1999) has suggested that the DEPI might be related to diagnoses of depression if certain moderator variables including Lambda, number of responses (R) and Erlebnistypus (EB) are taken into account. However, findings from recent studies do not support Viglione's position. In a study of depressed patients and the DEPI, Jansak (1996/1997) failed to find a moderating effect for either Lambda or R. Similarly, Krishnamurthy and Archer (2000) failed to find a moderating effect for EB.

\section{AIM OF THE STUDY}

Keeping all these views in mind, the current paper has been decided to be conducted. The main aim of the present study is to examine the difference of the Rorschach variables between depressive groups and normal controls, to examine the diagnostic efficacy of DEPI and to assess any correlation between the BDI scores and DEPI.

\section{HYPOTHESES}

There will be differences among the Rorschach variables between depressive groups and normal controls. DEPI will be able to diagnosis depressive patients according to ICD-10. 
A Study on Rorschach Depression Index in patients suffering from depression

\section{METHOD}

\section{PARTICIPANTS}

The sample consisted of 30 patients with depression diagnosed as per ICD-10 DCR criteria and of 30 normal control subjects selected from the outpatient psychiatry department of a reputed medical college of Kolkata. Participants were selected through purposive sampling technique.

\section{INCLUSION CRITERIA}

- Patients diagnosed with depression according to ICD-10 (DCR)

- Patients between the age range of 18-60 years

- Both males and females were included

- Protocols having a minimum of 14 responses

- For normal control subjects, score of less than 2 on GHQ will be included

- Participants who are able to comprehend test instructions

- Participants who are cooperative and giving consent for the study

\section{EXCLUSION CRITERIA}

- Patients with any co-morbid psychiatricillness or any other neurological or sensory impairment.

\section{INSTRUMENTS}

Socio Demographic and Clinical data sheet: A self prepared semi-structured data sheet was used for the patient and the control group. It was divided into socio-demographic details for both the groups and clinical data for the patient group. The sociodemographic variables were name age, sex, marital status, education, occupation, income. The variables in clinical data were duration of illness, diagnosis, duration of treatment, age of onset, mode of onset.

Beck Depression Inventory (BDI) : The Beck Depression Inventory (BDI), created by Aaron T. Beck, is a 21-question multiple-choice self-report inventory, one of the most widely used psychometric tests for measuring the severity of depression first published in 1961. The respondents were instructed to endorse how they had been feeling during the preceding two weeks. The internal consistency for the BDI-IA was good, with a Cronbach's alpha coefficient of around 0.85 , meaning that the items on the inventory are highly correlated with each other. Each question has a set of at least four possible responses, ranging in intensity. When the test is scored, a value of 0 to 3 is assigned for each answer and then the total score is compared to a key to determine the depression's severity. Higher total scores indicate more severe depressive symptoms.

Rorschach Psychodiagnostics: Rorschach Inkblot test was developed by Hermann Rorschach in 1921. This test was reportedly designed to reflect unconscious parts of the personality that "project" onto the stimuli. It measures functioning across a wide range of indices like perceptual, cognitive, affective, interpersonal and others. It consists of 10 cards with bilaterally symmetrical inkblots on each of them, of which five cards are of achromatic nature, two are of black and red ink, three are chromatic on a white background. It is one of the most popular instruments among the clinicians. Lubin et al., 1984) and also among researches, with over 6000 studies published by early 1980 (Aiken, 1993). The utility of this instrument in quantitative research has been improved by the work of Exner, whose Comprehensive System (Exner, 1991, 1993) has merged the several, older, competing methods of scoring into one uniform systematic and empirically validated system. Inter scorer reliabilities of 0.85 or higher. Early meta-analyses indicated that validity ranged from 0.40 to 0.50 .

General Health Questionnaire-12 (GHQ-12) : General Health Questionnaire (GHQ) was designed to be a self administered screening test aimed at detecting psychiatric disorders among respondents in community settings and non-psychiatric clinical settings such as primary care or among general medical out-patients. GHQ-12 is a short version of the general health questionnaire (GHQ). The cronbach's 
alpha for GHQ-12 is 0.82 and principal component analysis yielded three significant components accounting for 62 percent of variance. This test has been used as a screening tool for the assessment of psychiatric morbidity in normal samples. Each item is scored on four probable answers such as not at all, no more than usual, rather more than usual and much more than usual. Subject has to choose the most suitable one; each positive answer is scored 1 . Score ranges from 0 to 12 and 2 is cut-off.

\section{PROCEDURE}

After having informed consent, patients of depression group and normal controls were selected. Depression patients were included as per the ICD-10 DCR criteria. Normal control subjects were screened with the help of General Health Questionnaire (GHQ-12). Information about socio demographic data and clinical details was collected by using the socio demographic and clinical data sheet from the drawn sample. After that BDI and Rorschach Psycho-diagnostic Test was administered individually to all the participants to assess the personality structure. Rorschach protocols were scored using Exner Comprehensive System.

\section{STATISTICAL ANALYSIS}

Scores thus obtained were analyzed with Statistical Package for Social Sciences (SPSS-17). Descriptive statistics (percentages and mean) were used to describe demographics and clinical details of the participants and group differences were examined with Chi-Square test for categorical variables and t-test were used to compare the groups of depressive patients and normal control subjects. Diagnostic efficacy of DEPI was estimated by calculating sensitivity, specificity, positive and negative predictive value (Jogensen, et al.,2000). Sensitivity refers to the proportion of those with depression, according to ICD-10, who are correctly identified by DEPI. Specificity refers to the proportion of those without depression and were correctly identified so by DEPI. Positive predictive value refers to the probability that an individual has depression given the DEPI positive. Negative predictive value refers to the probability that an individual does not have depression given the DEPI negative.

\section{RESULTS \& DISCUSSION}

Depression patients and normal control subjects were matched with respect to age, sex, education, marital status, domicile where no significant difference has been found among these variables between depression patients and normal control subjects. It has also been observed that majority of the depressive patients were unemployed (43.3\%) due to illness because the patients could not manage their occupation for their severity of behavioural, affective and cognitive symptoms even at their workplace. In the group of normal control subject majority were employed (50\%) and the remaining were mostly housewife and students $(50 \%)$ who are able to manage their occupation and daily activities properly. The difference has been found to be significant at 0.01 level. The age of onset of depressive patient ranges from 20 years to 40 years with a mean of 32.70 and standard deviation of 4.79 . In case of mode of onset, majority of the patients $(50 \%)$ were having insidious mode of onset, whereas (46.7\%) were of acute mode and (3.3\%) having abrupt mode of onset.

Table 1 shows the comparison of DEPI variables among depressive groups and normal control group. Significant differences have been found on the five variables out of seven variables, of which four were found to be significant at 0.01 level and the remaining one at 0.05 level. The differences in the variables among the two groups which represents affect disturbances and low self esteem, inferiority feelings, negative views. This is in consistent with the findings of earlier studies by Dubey, 1989, Exner, 2003, Hartmann, 2003 where they found that the depressed patients differed significantly from the normal control subjects.

Table 2 shows the correlation between the BDI scores and DEPI among the depressive group. It is found that there is a negative correlation between the two variables which is not significant 
A Study on Rorschach Depression Index in patients suffering from depression

Table 1 : Comparison of Depression patients and Normal control subjects on DEPI criteria

\begin{tabular}{|c|c|c|c|c|c|}
\hline $\begin{array}{l}\text { Subjects } \\
\text { Rorschach } \\
\text { Variables }\end{array}$ & & $\begin{array}{c}\text { Depression } \\
\text { Patients }(\mathrm{N}=30) \\
N(\%)\end{array}$ & $\begin{array}{c}\text { Normal Control } \\
\text { Subjects }(\mathrm{N}=30) \\
\quad N(\%)\end{array}$ & df & $x^{2}$ value \\
\hline $\mathrm{FV}+\mathrm{VF}+\mathrm{V}>0$ or $\mathrm{FD}>2$ & $\begin{array}{c}\mathrm{FV}+\mathrm{VF}+\mathrm{V}>0 \\
\mathrm{FV}+\mathrm{VF}+\mathrm{V}<0 \\
\mathrm{FD}>2 \\
\mathrm{FD}<2\end{array}$ & $\begin{array}{c}20(33.3 \%) \\
5(8.3 \%) \\
5(8.3 \%) \\
0(0 \%)\end{array}$ & $\begin{array}{c}7(11.7 \%) \\
23(38.3 \%) \\
0(0 \%) \\
0(0 \%)\end{array}$ & 2 & $22.83^{* *}$ \\
\hline Color-Shading Blends $>0$ or $\mathrm{S}>2$ & $\begin{array}{l}\text { Color-Shading Blends }>0 \\
\text { Color-Shading Blends }<0 \\
\qquad \begin{array}{l}\mathrm{S}>2 \\
\mathrm{~S}<2\end{array} \\
\end{array}$ & $\begin{array}{c}7(11.7 \%) \\
23(38.3 \%) \\
0(0 \%) \\
0(0 \%) \\
\end{array}$ & $\begin{array}{c}3(5.0 \%) \\
27(45 \%) \\
0(0 \%) \\
0(0 \%)\end{array}$ & 1 & $11.28^{* *}$ \\
\hline $\begin{array}{l}\{3(\mathrm{rF}+\mathrm{Fr})+\operatorname{Sum}(2)\} / \mathrm{R}>0.44 \text { and } \\
\mathrm{rF}+\mathrm{Fr}=0 \text { or } \\
\{3(\mathrm{rF}+\mathrm{Fr})+\operatorname{Sum}(2)\} / \mathrm{R}<0.33\end{array}$ & $\begin{array}{c}\{3(\mathrm{rF}+\mathrm{Fr})+\operatorname{Sum}(2)\} / \mathrm{R}>0.44 \text { and } \\
\mathrm{rF}+\mathrm{Fr}=0 \\
\{3(\mathrm{rF}+\mathrm{Fr})+\operatorname{Sum}(2)\} / \mathrm{R}<0.44 \text { and } \\
\mathrm{rF}+\mathrm{Fr}>0 \\
\{3(\mathrm{rF}+\mathrm{Fr})+\operatorname{Sum}(2)\} / \mathrm{R}<0.33 \\
\{3(\mathrm{rF}+\mathrm{Fr})+\operatorname{Sum}(2)\} / \mathrm{R}>0.33\end{array}$ & $\begin{array}{c}14(23.3 \%) \\
12(20 \%) \\
4(6.7 \%) \\
0(0 \%)\end{array}$ & $\begin{array}{c}9(15 \%) \\
21(35 \%) \\
0(0 \%) \\
0(0 \%)\end{array}$ & 2 & $7.52^{*}$ \\
\hline Afr $<0.46$ or Blends $<4$ & $\begin{array}{l}\text { Afr }<0.46 \\
\text { Afr }>0.46 \\
\text { Blends }<4 \\
\text { Blends }>4\end{array}$ & $\begin{array}{c}17(28.3 \%) \\
5(8.3 \%) \\
8(13.3 \%) \\
0(0 \%)\end{array}$ & $\begin{array}{c}12(20 \%) \\
18(30 \%) \\
0(0 \%) \\
0(0 \%)\end{array}$ & 2 & $16.21^{* *}$ \\
\hline $\begin{array}{l}\text { SumShading }>\mathrm{FM}+\mathrm{m} \text { or } \\
\text { SumC'>2 }\end{array}$ & $\begin{array}{c}\text { SumShading }>\mathrm{FM}+\mathrm{m} \\
\text { SumShading }<\mathrm{FM}+\mathrm{m} \\
\text { SumC'>2 } \\
\text { SumC } C^{\prime}<2\end{array}$ & $\begin{array}{c}8(13.3 \%) \\
21(35 \%) \\
1(1.7 \%) \\
0(0 \%)\end{array}$ & $\begin{array}{c}4(6.7 \%) \\
26(43.3 \%) \\
0(0 \%) \\
0(0 \%)\end{array}$ & 2 & $14.05^{* *}$ \\
\hline $\mathrm{MOR}>2$ or $(2 * \mathrm{AB}+\mathrm{Art}+\mathrm{Ay})>3$ & $\begin{array}{c}\mathrm{MOR}>2 \\
\mathrm{MOR}<2 \\
(2 * \mathrm{AB}+\mathrm{Art}+\mathrm{Ay})>3 \\
(2 * \mathrm{AB}+\mathrm{Art}+\mathrm{Ay})<3\end{array}$ & $\begin{array}{c}8(13.3 \%) \\
17(28.3 \%) \\
4(6.7 \%) \\
1(1.7 \%)\end{array}$ & $\begin{array}{c}2(3.3 \%) \\
28(46.7 \%) \\
0(0 \%) \\
0(0 \%)\end{array}$ & 3 & $1.92 \mathrm{~ns}$ \\
\hline $\begin{array}{l}\mathrm{COP}<\mathrm{C} 2 \text { or } \\
\left(\mathrm{Bt}+2^{*} \mathrm{Cl}+\mathrm{Ge}+\mathrm{Ls}+2 * \mathrm{Na}\right) / \mathrm{R}>0.24\end{array}$ & $\begin{array}{c}\mathrm{COP}<\mathrm{C} 2 \\
\mathrm{COP}>\mathrm{C} 2 \\
\left(\mathrm{Bt}+2^{*} \mathrm{Cl}+\mathrm{Ge}+\mathrm{Ls}+2^{*} \mathrm{Na}\right) / \mathrm{R}>0.24 \\
\left(\mathrm{Bt}+2^{*} \mathrm{Cl}+\mathrm{Ge}+\mathrm{Ls}+2^{*} \mathrm{Na}\right) / \mathrm{R}<0.24\end{array}$ & $\begin{array}{c}17(28.3 \%) \\
5(8.3 \%) \\
6(10 \%) \\
2(3.3 \%)\end{array}$ & $\begin{array}{c}14(23.3 \%) \\
16(26.7 \%) \\
0(0 \%) \\
0(0 \%)\end{array}$ & 3 & $2.86 \mathrm{~ns}$ \\
\hline
\end{tabular}

${ }^{*} \mathrm{p}<0.05$ level, ${ }^{* *} \mathrm{p}<0.01$ level

statistically. This is in agreement with the previous studies done by Fisha, 2001 where a low and statistically non significant correlation was found between BDI scores and DEPI. It is also found to correlate very poorly with other objective measures of depression like BDI (Campbell \& Lorandos, 2010).

Table 3 shows the efficacy of depression index (DEPI) of Rorschach Inkblot Test. With cut off five, the positive predictive value (PPV) was found to be
0.76 , negative predictive value (NPV) was found to be 0.58 . Sensitivity was found to be 0.66 and specificity was found to be 0.80 . It can be said from the table that the negative predictive value and sensitivity is low. In terms of positive predictive value, the probability of an individual to have depression when the DEPI is positive is low. The proportion of those with depression correctly identified by DEPI is also found to be low. Thus it is apparent that the diagnostic efficacy of 
A Study on Rorschach Depression Index in patients suffering from depression

Table 2 : Correlation between BDI scores and DEPI of Depressive group

\begin{tabular}{|c|c|}
\hline Variables (Depressive Group) & Depression Index (DEPI) \\
\hline Beck Depression Inventory (BDI) & $0.43 \mathrm{~ns}$ \\
\hline
\end{tabular}

Table 3 : Efficacy of Depression Index (DEPI) of Rorschach Inkblot Test

\begin{tabular}{|c|c|c|c|c|}
\hline $\begin{array}{c}\text { Perceptual } \\
\text { Thinking Index } \\
\text { (PTI) }\end{array}$ & $\begin{array}{c}\text { Positive Predictive } \\
\text { Value (PPV) }\end{array}$ & $\begin{array}{c}\text { Negative Predictive } \\
\text { Value (NPV) }\end{array}$ & Sensitivity & Specificity \\
\hline 5 & 0.76 & 0.58 & 0.66 & 0.80 \\
\hline
\end{tabular}

DEPI is very low for diagnosing patients with depression. This finding is in consistent with other studies that have suggested that the DEPI has limited ability to diagnose depression (Greenwald, 1997; Ritsher, Slivko-Kolchik \& Oleichik, 2001).

\section{CONCLUSION}

DEPI scores alone should not suggest the diagnosis of depression and ideally the scores should be interpreted in conjunction with the history and findings on the mental status examination. DEPI scores should be interpreted with considerable caution when applied for diagnostic purposes. Previous studies have concluded that the variables in Depression Index are typically not directly related to observable behaviour, so it should not be used on its own to diagnose a major depressive disorder. Instead, the DEPI serves to identify individuals who may be emotionally distraught, cognitively pessimistic, lethargic or self-defeating. Positive values

of DEPI are probably been interpreted as representing an affective problem. Future studies may focus on evaluating DEPI against individual Rorschach variables that are useful in detecting depressive features.

\section{REFERENCES}

Exner,J.E.Jr.(1993). The Rorschach:AComprehensive System. Vol 1 Basic Foundations ( $3^{\text {rd }}$ ed.). New York: Wiley.

Exner,J.E.Jr.(1991).TheRorschach:A Comprehensive System. Vol 2. Interpretation ( $2^{\text {nd }}$ ed.). New York: Wiley.

Ganellen, R.J. (1996a). Comparing the diagnostic efficiency of the MMPI, MCMI-II, and Rorschach : A review. Journal of Personality Assessment, 67, 219-243.

Ganellen, R.J. (1996b). Integrating the Rorschach and the MMPI-2 in personality assessment. Mahwah, NJ : Lawrence Erlbaum Associates.

Lipovsky, J.A., Finch, A.J., \& Belter, R.W. (1989). Assessment of depression in adolescents : Objective and projective measures. Journal of Personality Assessment, 53, 449- 458.

Archer, R.P., \& Krishnamurthy, R. (1997). MMPI-A and Rorschach indices related to depression and conduct disorder : An evaluation of the incremental validity hypothesis. Journal of Personality Assessment, 69, 517-533.

Carlson, C.F., Kula, M.L., \& St. Laurent, C.M. (1997). Rorschach revised DEPI and CDI with inpatient 
A Study on Rorschach Depression Index in patients suffering from depression

major depressives and borderline personality disorder with major depression: Validity issues. Journal of Clinical Psychology, 53, 51-58.

Carter, C.L., \& Dacey, C.M. (1996). Validity of the Beck Depression Inventory, MMPI, and Rorschach in assessing adolescent depression. Journal of Adolescence, 19, 223-231.

Ball, J.D., Archer, R.P., Gordon, R.A., \& French, J. (1991). Rorschach depression indices with children and adolescents: Concurrent validity findings. Journal of Personality Assessment, 57, 465- 476 .

Viglione, D.J. (1999). A review of recent research addressing the utility of the Rorschach. Psychological Assessment, 11, 251-265.

Jansak, D.M. (1997). The Rorschach Comprehensive System Depression Index, depression heterogeneity, and the role of self-schema (Doctoral dissertation, California School of Professional Psychology, San Diego, 1996). Dissertation Abstracts International, 57, 6576B.

Krishnamurthy, R., \& Archer, R.P. (2000). An evaluation of the effects of Rorschach EB style on the diagnostic utility of the Depression Index. Manuscript submitted for publication.
Exner, J.E. (2003). The Rorschach: A Comprehensive System: Vol 1. Basic Foundations ( $4^{\text {th }}$ ed.). Hoboken, NJ : Wiley.

Hartmann, E., Wang, C.E., Berg, M., \& Saether, L. (2003). Depression and vulnerability as assessed by the Rorschach method. Journal of Personality Assessment, 81(3), 242-255.

Campbell, T.W. E Lorandos, D. (2010). Cross-examining experts in the behavioral sciences. St. Paul, MN : Thomson-West.

Dubey, B.L. (1989). Rorschach profile of Indian executives, Indian Journal of Clinical Psychology, 16, 74-76.

Greenwald, D.F. (1997). Comparison between the Rorschach depression index and depression related measures in a non patient sample. Psychological Reports, 80 (Pt. 2, 3), 1151-1154.

Ritsher, J.B., Slivko-Kolchik, E, B., \& Oleichik, I.V. (2001). Assessing depression in Russian psychiatric patients: Validity of MMPI and Rorschach. Assessment, 8(4), 373-389 